\title{
Papillär-zystischer Pankreastumor: Eine seltene Erkrankung junger Mädchen
}

\author{
S. Lange, H. G. Peitz, M. W. Kellner, E. Waltermann \\ Radiologische Abteilung, Kinderkrankenhaus der Stadt Köln
}

\section{Zusammenfassung}

Papillär-zystische Pankreastumoren sind im Kindesalter sehr selten. Sie treten bei Mädchen häufiger auf als bei Jungen. Die Tumorart wurde erstmals 1959 beschrieben. Nach seiner Erstbeschreiberin wird der Tumor auch als Frantztumor bezeichnet. Der Malignitätsgrad des Tumors ist niedrig und eine Metastasierung selten. Die Prognose der Erkrankung ist nach vollständiger Tumorresektion gewöhnlich günstig. Es ist deshalb wichtig, zwischen einem papillär-zystischen Pankreastumor und anderen Pankreastumoren zu unterscheiden. Im Folgenden berichten wir über eine 12-jährige Patientin mit einem papillär-zystischen Pankreastumor, um die klinischen und radiologischen Charakteristika dieses Tumors aufzuzeigen.

Papillar-cystic tumor of pancreas: A rare disease of adolescent females

Papillary-cystic tumors of the pancreas, so-called Frantz tumors, are very rare. They occur mainly in adolescent and young adult females.The tumors are neoplasms of low malignancy with infrequent metastases. After complete surgical resection the prognosis is usually excellent. For that reason it is important to make the differential diagnosis berween papillary-cystic tumor of pancreas and other pancreatic tumors. We describe a case of the papillary-cystic tumor of the pancras in a12-year-old girl to illustrate the clinical and radiological characteristics of this tumor.

\section{Fallbericht}

Anamnese: Stationäre Aufnahme des 12-jährigen Mädchens in eine auswärtige Klinik wegen eines zunehmenden Ikterus, wechselnder Übelkeit und acholer Stühle, Verdachtsdiagnose: Hepatitis.

Untersuchungsbefund: Leicht reduzierter Allgemein- und Ernährungszustand, geringer Ikterus, ca. $8-10 \mathrm{~cm}$ großer derber rechtsseitig tastbarer Oberbauchtumor.

Laborbefunde: Im Sinne einer Cholestase und Pankreatitis verändert.

Bildgebende Diagnostik: MRT (Abb.1) und MRCP (Abb.2): ca. $12 \mathrm{~cm}$ großer, gekapselter, teilvaskularisierter, fettgewebsreicher, solider Tumor in der Leberpforte bzw. im Pankreaskopfbereich mit konsekutiver Erweiterung der intra- und extrahepatischen Gallenwege, unauffälliger Ductus pancreaticus, keine Infiltration in umgebende Organe, nach Kontrastmittelgabe Signalverstärkung der Tumorkapsel.

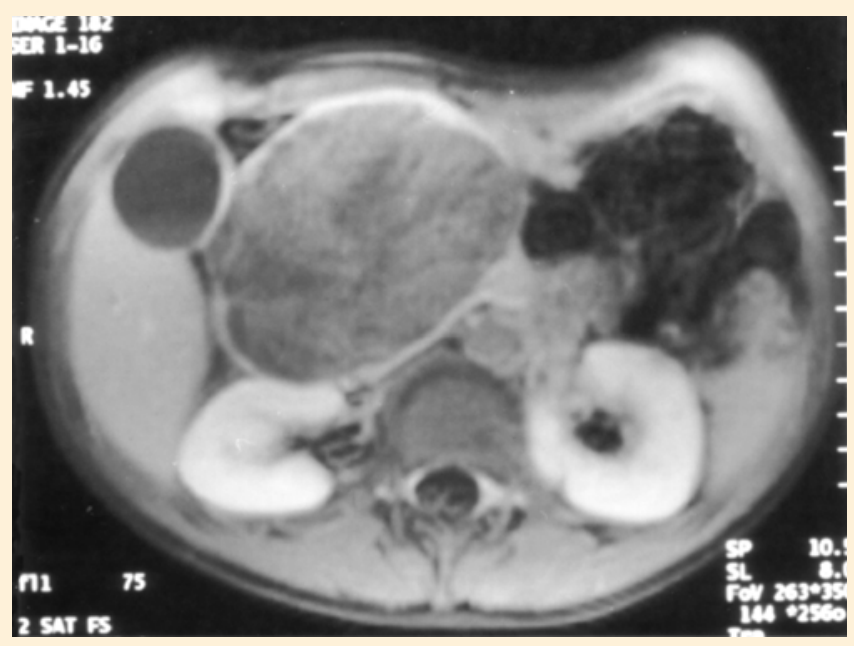

Abb. 1 MRT (Gad-DTPA i.v., T1 w FLASH 2D).
Weiterer Verlauf: Nach dieser Diagnostik Verlegung des Mädchens zur weiteren Abklärung und der sich daraus ergebenden Therapie in unser Haus. Vor der Laparotomie mit geplanter Biopsie nochmals Durchführung einer Abdomensonographie (Abb.3). Diese erbringt gegenüber den Voruntersuchungen keine neuen Erkenntnisse. Eine Magen-Darm-Passage zeigt die Verlagerung des Duodenums durch den Tumor (Abb. 4). Durch eine Laparatomie mit gezielter Biopsie kann der Tumor endgültig dem Pankreas zugeordnet und die Diagnose papillär-zystischer Pankreastumor gestellt werden (Prof. Dr. Dr. h.c. Harms, Institut für Pathologie, Universitätsklinik Kiel, 100280/00 ).

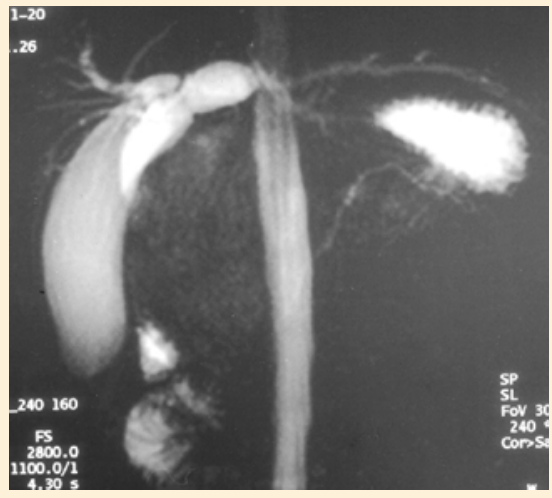

Abb. 2 MRCP (T2 w, fettunterdrückt)

* Für die Überlassung der MRT-Bilder danken wir Herrn Prof. Dr. med. R. Janson, Institut für Radiologie und Nuklearmedizin, Klinikum Leverkusen $\mathrm{GmbH}$.

Therapie: Komplette Pankreaskopfresektion mit pyloruserhaltender Gastroduodenostomie, bilidigestiver Anastomose mit antirefluxiver Mukosaklappe und y-Roux-Pancreaticojejunostomie mit Cholezystektomie. Das Operationspräparat zeigt keinen Befall der entnommenen Lymphknoten sowie tumorfreie Resektat- und Pankreasresektionsränder. Von daher kann eine komplette Entfernung des Tumors angenommen werden. 


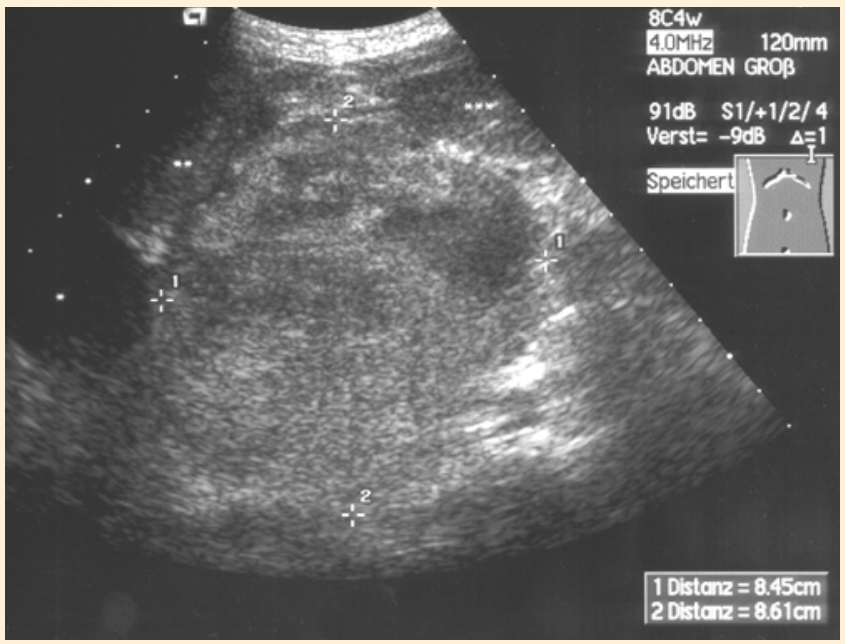

Abb. 3 Sonographie (4 MHz).

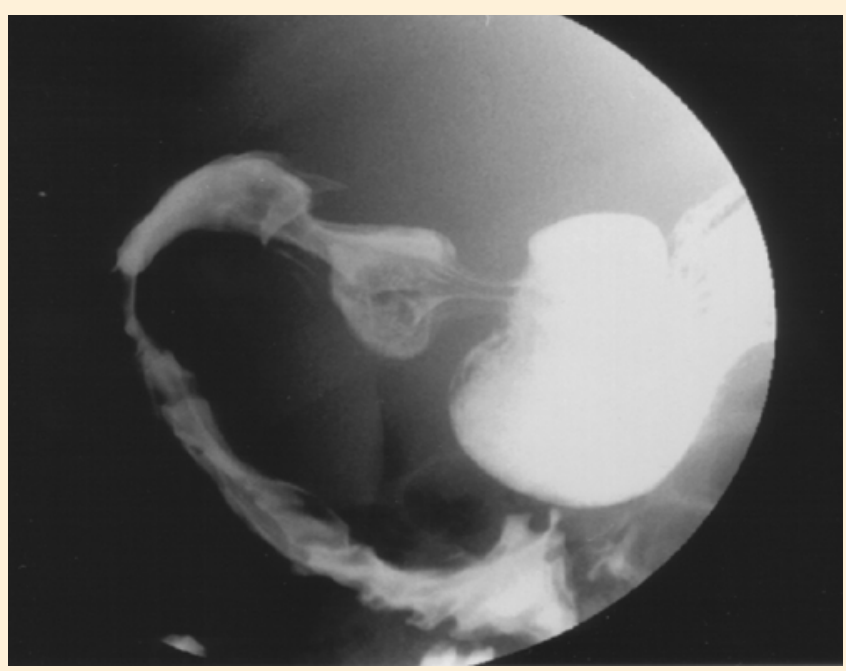

Abb. 4 Magen-Darm-Passage.

\section{Kommentar}

Der papillär-zystische Tumor des Pankreas ist eine niedrigmaligne Neoplasie. Er kommt am häufigsten bei Frauen zwischen dem 20. und 30. Lebensalter vor. Das Auftreten im Kindesalter ist sehr selten. Bis 1999 wurden 62 Fälle, davon 57 Mädchen und 5 Jungen, bekannt [1]. Die Klinik ist untypisch. Lange Zeit kann der Tumor symptomlos zu einer großen abdominalen Raumforderung heranwachsen. Häufig wird er erst entdeckt, wenn er einen Durchmesser von durchschnittlich $10 \mathrm{~cm}$ hat und die verdrängende Wirkung des Tumors das klinische Bild - z.B. Bauchschmerzen, Brechreiz, Appetitlosigkeit, Ikterus, Durchfall, tastbarer Tumor - bestimmt. Der Tumor ist durch ein nicht invasives Wachstum und eine seltene Metastasierung, meist in die Leber oder das Peritoneum, charakterisiert. Die metastatische Tumorausdehnung wird durch Bauchtraumen, die den Tumor mitbetreffen, oder durch Tumorbiopsien begünstigt [2].

Die komplette Tumorresektion ist die adäquate Behandlung. Die Prognose ist danach sehr gut.
In der Bildgebung - Sonographie, CT, MRT, MRCP - hat der Tumor ein breites Erscheinungsbild. Obgleich nicht spezifisch, zeigt er sich als gut abgekapselte solide Masse mit zentralen zystischen Arealen unterschiedlicher Ausdehnung. Dabei korrespondieren die zystischen Räume mit hämorrhagischen Nekrosen im histologischen Befund.

Gelegentlich werden randständige Verkalkungen gefunden [3] Der Ursprung des Tumors kann sowohl mit der Sonographie als auch der CT oder der MRT gut abgeklärt werden. Die MRT in Kombination mit der MRCP ist wegen der sehr guten Bildqualität bei der Darstellung des Oberbauches zur Beurteilung der Pankreasloge, zur Differenzierung der hämorrhagischen Bezirke in den soliden Formationen, zur Beurteilung des Gallengangs- und Pankreasgangsystems und zur Klärung der Tumorinvasion günstig. Der Pankreasgang ist meist bogig verlagert bzw. ausgezogen, aber entgegen den Befunden, die man bei hoch malignen Pankreastumoren findet, nicht stenosiert. Beim Druck auf das Gallengangssystem kann es zu Abflussstörungen mit entsprechenden Komplikationen kommen. Eine Infiltration in die Nachbarorgane ist sehr selten [4].

Da Pankreastumoren sich in allen heute zur Verfügung stehenden diagnostischen Verfahren unspezifisch darstellen, ist eine zuverlässige Differenzierung noch nicht möglich.

Übereinstimmend mit der Literatur weisen in unserem Fall aber folgende Kriterien auf die Diagnose papillär-zystischer Pankreastumor hin:

1) das Alter

2) das Geschlecht

3) der große palpable Oberbauchtumor mit kurzer klinischer Anamnese

4) der durch die Bildgebung festgestellte solid zystische, gut abgekapselte, in der Kapsel Kontrastmittel aufnehmende Pankreastumor ohne Infiltration in die umgebenden Organe und ohne Hinweis auf Metastasierung, der zwar zur Stauung des Gallengangssystems führt, aber keine Malignitätszeichen am Pankreasgangsystem erkennen lässt.

Sind diese Kriterien bei dem Vorliegen eines Pankreastumors erfüllt, ist es unseres Erachtens möglich, eine diagnostische und prognostische Aussage zu treffen und die Patientinnen einer operativen kurativen Therapie zuzuführen.

\section{Literatur}

1 Soudack M et al. Harefuah 2000; 138: 105-107

${ }^{2}$ Levy P et al. Gastroenterol Clin Biol 1997; 21: 789 - 793

3 Poustchi-Amin M et al. Pediatr Radiol 1995; 25: 509-511

${ }^{4}$ Siech M et al. Chirurg 1996; 67: $1012-1015$

\section{Dr. med Sabine Lange}

Kinderkrankenhaus der Stadt Köln

Amsterdamer Straße 59

50735 Köln 